\title{
A New Generalized Cauchy Distribution with an Application to Annual One Day Maximum Rainfall Data
}

\author{
Cory Ball ${ }^{1}$, Binod Rimal ${ }^{1}$, Sher Chhetri ${ }^{2, *}$ \\ ${ }^{1}$ Department of Mathematical Sciences, Florida Atlantic University, Boca Raton, FL, USA \\ ${ }^{2}$ Division of Science, Mathematics, and Engineering, University of South Carolina, Sumter, SC, USA
}

\begin{abstract}
In this article, we introduce a new three-parameter transmuted Cauchy distribution using the quadratic rank transmutation map approach. Some mathematical properties of the proposed model are discussed. A simulation study is conducted using the method of maximum likelihood estimation to estimate the parameters of the model. We use two real data sets and compare various statistics to show the fitting and versatility of the proposed model.
\end{abstract}

Keywords Cauchy distribution, transmuted Cauchy distribution, Quadratic rank transmuted map, Maximum likelihood estimation, Hazard rate function.

AMS 2010 subject classifications 60E05, 62P99

DOI: $10.19139 /$ soic-2310-5070-1000

\section{Introduction}

The Cauchy distribution, named after Augustin Cauchy (1789 -1857), is a stable familiy of distributions in which the expected value and variance do not exist. Stable distributions are a special family of probability distributions appropriate for modeling data that are heavy tailed and skewed. By Kateregga et al. [22], probability densities of stable distributions do not have closed-form representations except for the case of a Gaussian, Cauchy and Inverse Gaussian or Pearson distributions. There are some generalizations of the Cauchy distribution using different frameworks, for instance, [7], [13], among others. A detailed study of the generalized Cauchy family of distributions with applications has been studied by Alzaatreh et al. [7] in 2016 where authors use the framework proposed by Aljarrah et al. [2]. The Cauchy distribution has been used in many applications such as mechanical and electrical theory, physical anthropology, measurement problems, risk and financial analysis, Alzaatreh et al. [7].

The probability density function (PDF) of the Cauchy distribution with location parameter $x_{0} \in \mathbb{R}$ and the scale parameter $\gamma>0$ is given by

$$
g\left(x ; x_{0}, \gamma\right)=\frac{1}{\pi}\left[\frac{\gamma}{\left(x-x_{0}\right)^{2}+\gamma^{2}}\right]
$$

where $-\infty<x<\infty$.

The corresponding cumulative distribution function (CDF) of the Cauchy distribution is given by

$$
G\left(x ; x_{0}, \gamma\right)=\frac{1}{\pi} \arctan \left(\frac{x-x_{0}}{\gamma}\right)+\frac{1}{2} .
$$

\footnotetext{
*Correspondence to: Sher Chhetri (Email: schhetri@mailbox.sc.edu). Division of Science, Mathematics, and Engineering, University of South Carolina, Sumter: 200 Miller Road, Sumter, South Carolina, 29150, USA.
}

ISSN 2310-5070 (online) ISSN 2311-004X (print)

Copyright (C) 2021 International Academic Press 
Note that for $\gamma=1$ and $x_{0}=0$, equations (1) and (2) reduces to the PDF and the CDF of the standard Cauchy distribution

$$
g(x, 0,1)=\frac{1}{\pi\left(1+x^{2}\right)}
$$

and

$$
G(x ; 0,1)=\frac{\arctan (x)}{\pi}+\frac{1}{2}
$$

respectively, where $-\infty<x<\infty$.

A new approach of generalizing statistical distributions was proposed by Shaw et al. ([28],[29]). Their concept is defined by transmutation maps that are functional composition of the cumulative distribution function of one distribution with the quantile function of another distribution. Shaw et al. ([28],[29]) used quadratic rank transmuted map (QRTM) approach to construct flexible models by adding an additional parameter.

We consider a pair of distributions $F(x)$ and $G(x)$, where $G(x)$ is the baseline (or a sub-model) distribution of $F(x)$. A random variable $X$ is said to have a transmuted probability distribution with the CDF $F(x)$ if

$$
F(x)=(1+\lambda) G(x)-\lambda G(x)^{2},|\lambda| \leq 1,
$$

and the corresponding PDF $f(x)$ of the transmuted probability distribution is

$$
f(x)=g(x)(1+\lambda-2 \lambda G(x))
$$

where $g(x)$ is the PDF of the sub-model $G(x)$. We note that when $\lambda=0$, equations (3) and (4) reduces to the distribution of the baseline random variable.

Many new distributions and generalizations using transmutation map have been proposed after Aryal et al. [8] studied "On the transmuted extreme value distribution with application" in (2009). Some of them are the work by Ashour [11]: transmuted Lomax distribution, Merovci et al. [24]: transmuted Pareto distribution, Pal et al. [26]: beta transmuted Weibull distribution, Khan et al. [23]: transmuted Kumaraswamy distribution, Elbatal et al. [21]: transmuted Dagum distribution with applications, Chhetri et al. [16]: the beta transmuted Pareto distribution, Chhetri et al. [15]: the Kumaraswamy transmuted Pareto distribution, Aryal [9]: transmuted loglogistic distribution, Chesneau et al. [14]: a weighted transmuted exponential distribution with environmental applications, and others. Many researchers have proposed generalized probability distributions using various approaches. Some of the recent works are by Nadarajah et al. [25] : a skewed truncated Cauchy distribution with applications in economics, Cordeiro et al. [17]: the beta-half-Cauchy distribution, Aryal et al. [10]: on the beta exponential Pareto distribution, Alzaatreh et al. [5]: a new method for generating families of continuous distributions, Alshkaki [4]: a generalized modification of the Kumaraswamy distribution for modeling and analyzing real-life data, Alizadeh et al. [1]: a new two-parameter lifetime distribution: properties, applications and different method of estimations, Cordeiro et al. [18]: a new family of generalized distributions, and host of others.

In this work, we use Cauchy distribution to develop the transmuted Cauchy distribution using the framework proposed by Shaw et al. [[28], [29]]. The main contribution of this work is the introduction of a new distribution that outperforms its classical base distribution as well as other distributions in applications. This further demonstrates the need to investigate more general distributions used in engineering and scientific applications.

This paper is outlined as follows. In section 2, we formulate the transmuted Cauchy distribution. In section 3 , we discuss the quantile function and random number generation. Estimation of parameters of the distribution using the maximum likelihood method is discussed in section 4 and expressions for the hazard rate function and reversed hazard rate function are derived in section 5. Simulation studies and applications of the proposed model are presented in section 6 and section 7 respectively and, finally we provide the concluding remarks in section 8 . 


\section{The Transmuted Cauchy Distribution}

In this section, we use the transmutation map method proposed by Shaw et al. [[28], [29]] and derive the PDF and CDF of the generalization of the Cauchy distribution so-called "transmuted Cauchy distribution" (TCD).

Substituting (2) into (3), we obtain the CDF of the three parameter transmuted Cauchy distribution:

$$
\begin{aligned}
F\left(x ; x_{0}, \gamma, \lambda\right) & =-\frac{\lambda}{\pi^{2}}\left[\arctan \left(\frac{x-x_{0}}{\gamma}\right)\right]^{2}+\frac{1}{\pi} \arctan \left(\frac{x-x_{0}}{\gamma}\right) \\
& +\frac{\lambda}{4}+\frac{1}{2}
\end{aligned}
$$

where $x_{0} \in \mathbb{R}, \gamma>0,|\lambda| \leq 1$ and $x \in \mathbb{R}$.

Figures (1) and (2) show the graphical behavior of the CDF of transmuted Cauchy distribution for selected values of the parameters $x_{0}, \gamma$ and $\lambda$.

Similarly, the PDF of the transmuted Cauchy distribution may be written as

$$
f\left(x ; x_{0}, \gamma, \lambda\right)=\frac{\gamma}{\pi^{2}\left[\left(x-x_{0}\right)^{2}+\gamma^{2}\right]}\left[\pi-2 \lambda \arctan \left(\frac{x-x_{0}}{\gamma}\right)\right] .
$$

Figures (3) and (4) show the graphical behavior of the PDF of transmuted Cauchy distribution for selected values of the parameters $x_{0}, \gamma$ and $\lambda$.

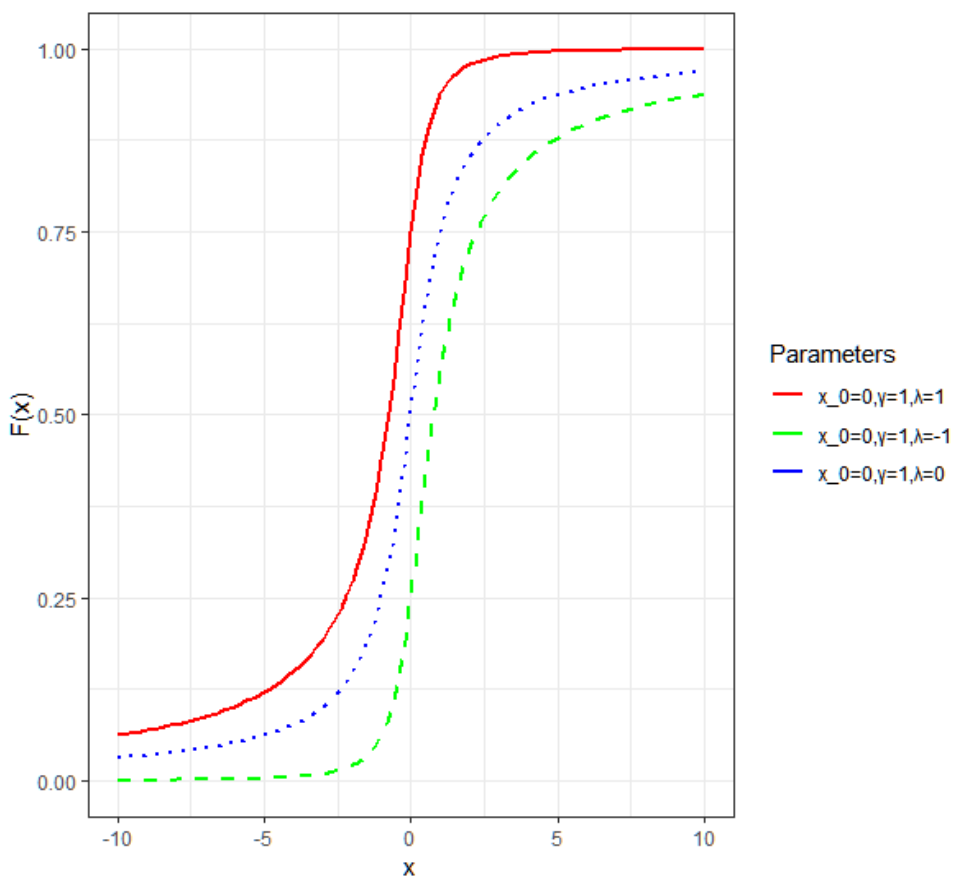

Figure 1. CDF plots of the transmuted Cauchy distribution with various parameters 


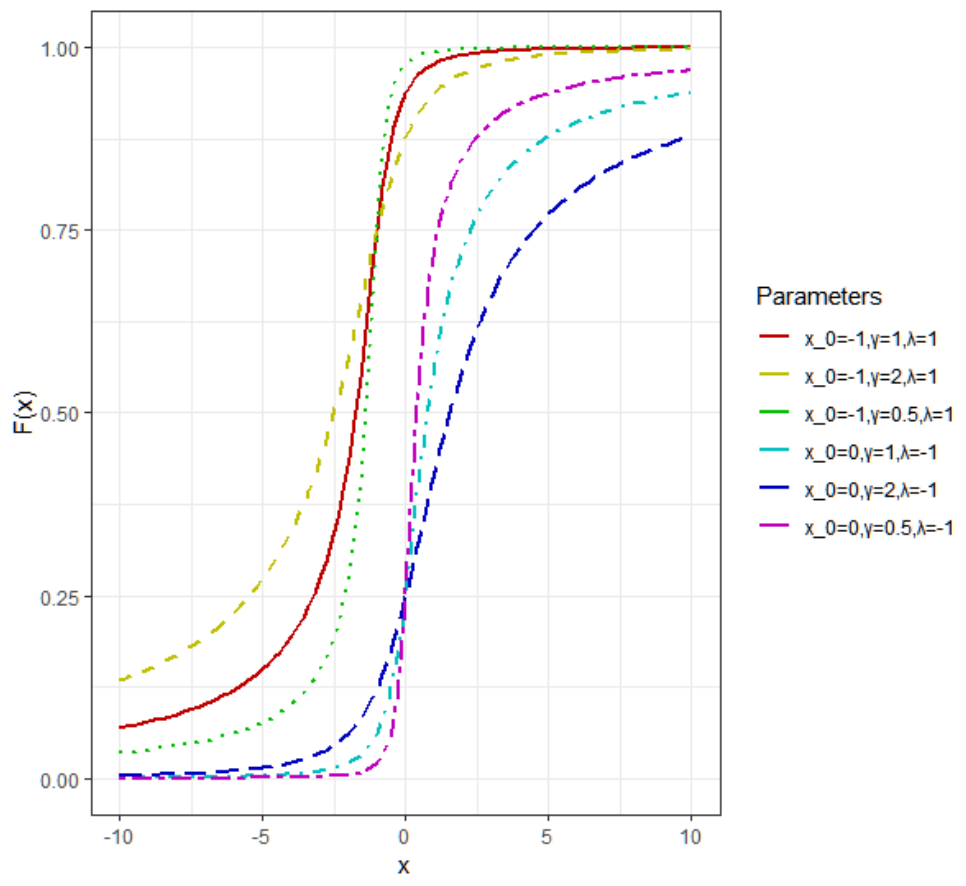

Figure 2. CDF plots of the transmuted Cauchy distribution with various parameters

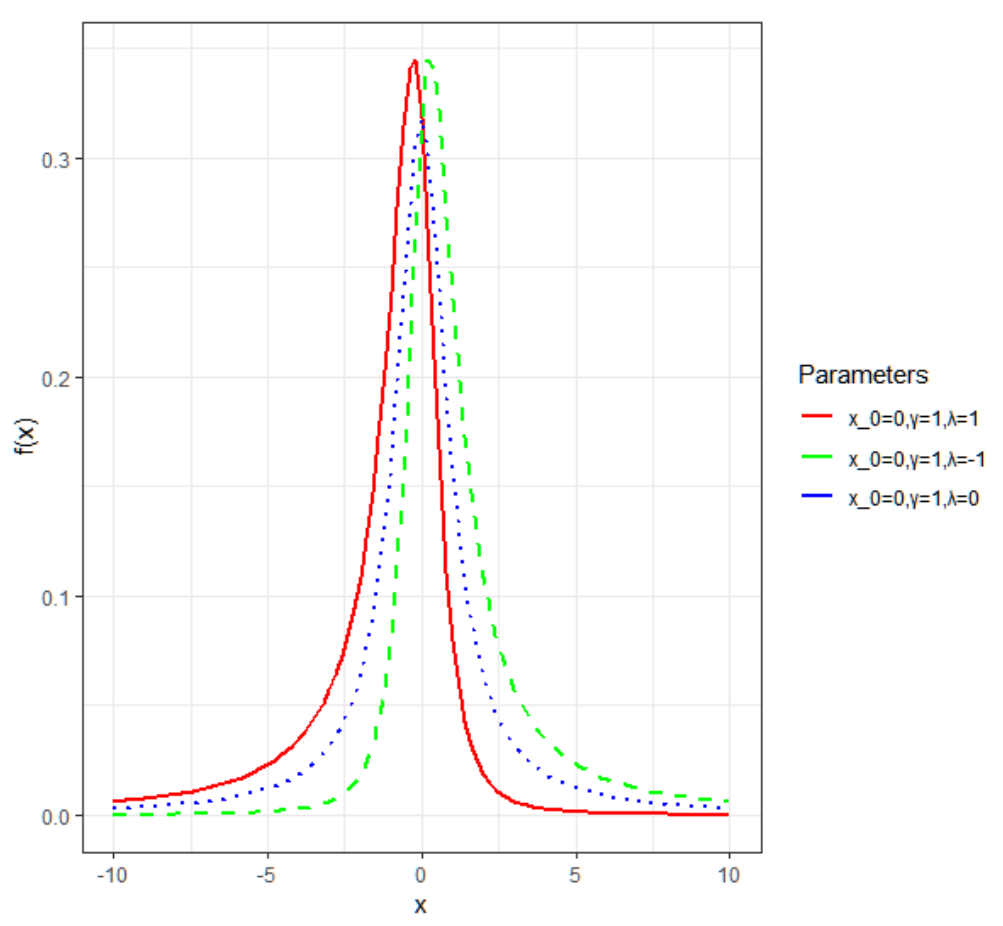

Figure 3. PDF plots of the transmuted Cauchy distribution with various parameters 


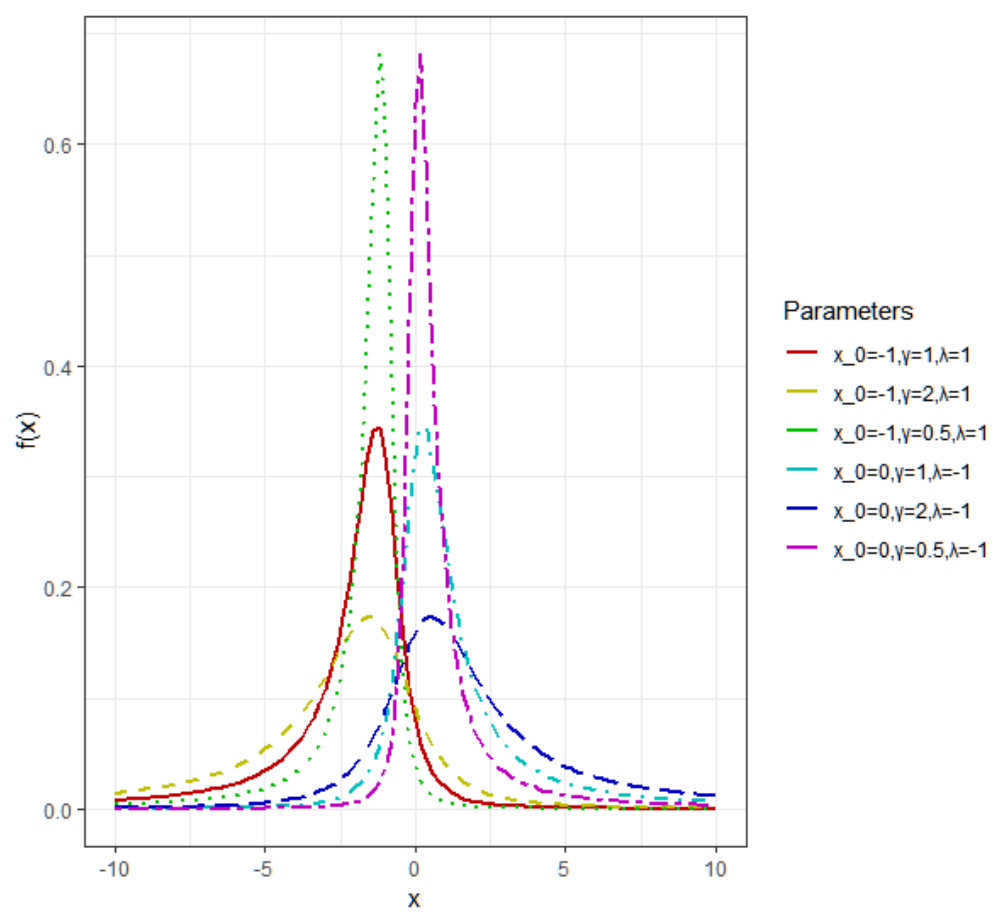

Figure 4. PDF plots of the transmuted Cauchy distribution with various parameters

\section{Quantile Function and Random Number Generator}

We use the inverse transformation method to generate random numbers form the transmuted Cauchy distribution. The $q$ th quantile, $x_{q}$, of the TCD is defined as the real solution of

$$
q=\operatorname{Pr}\left(X \leq x_{q}\right)=F\left(x_{q}\right), 0 \leq q \leq 1,
$$

where $F($.$) is given by (5).$

Thus, we obtain

$$
q=-\frac{\lambda}{\pi^{2}}\left[\arctan \left(\frac{x_{q}-x_{0}}{\gamma}\right)\right]^{2}+\frac{1}{\pi} \arctan \left(\frac{x_{q}-x_{0}}{\gamma}\right)+\frac{\lambda+2}{4},
$$

which is quadratic in $\arctan \left(\frac{x_{q}-x_{0}}{\gamma}\right)$. Then $x_{q}$ is the real solution of the equation

$$
\arctan \left(\frac{x_{q}-x_{0}}{\gamma}\right)=\frac{\pi\left(1 \pm \sqrt{\lambda^{2}-4 \lambda q+2 \lambda+1}\right)}{2 \lambda} .
$$

Thus,

$$
x_{q}=x_{0}+\gamma \tan \left(\frac{\pi\left(1 \pm \sqrt{\lambda^{2}-4 \lambda q+2 \lambda+1}\right)}{2 \lambda}\right) .
$$

As $q \rightarrow 1^{-}$, we want $x_{q} \rightarrow \infty$ and as $q \rightarrow 0^{+}$, we want $x_{q} \rightarrow-\infty$. So for $\lambda \neq 0$, we have

$$
x_{q}=x_{0}+\gamma \tan \left(\frac{\pi\left(1-\sqrt{\lambda^{2}-4 \lambda q+2 \lambda+1}\right)}{2 \lambda}\right) .
$$

For simulation studies, we will choose specific values of parameters $x_{0}, \gamma, \lambda$ and $q \in(0,1)$, and use expression (9) to generate a random variable $X$ having the transmuted Cauchy distribution (5). 


\section{Parameter Estimation}

There are several approaches for parameter estimation. The most commonly used method is the maximum likelihood estimation method to estimate model parameters.

Let $X_{1}, X_{2}, \ldots, X_{n}$ be a random sample from the transmuted Cauchy distribution with observed values $x_{1}, x_{2}, \ldots, x_{n}$ and $\Theta=\left(x_{0}, \gamma, \lambda\right)^{T}$ be the parameter vector. The likelihood function of the transmuted Cauchy distribution is

$$
L(\Theta \mid x)=\frac{\gamma^{n}}{\pi^{2 n}} \prod_{i=1}^{n} \frac{\pi-2 \lambda \arctan \left(\frac{x_{i}-x_{0}}{\gamma}\right)}{\left(x_{i}-x_{0}\right)^{2}+\gamma^{2}} .
$$

So, the log-likelihood function can be written as

$$
\begin{aligned}
l(\Theta \mid x) & =n \log \gamma-2 n \log \pi+\sum_{i=1}^{n}\left[\log \left(\pi-2 \lambda \arctan \left(\frac{x_{i}-x_{0}}{\gamma}\right)\right)\right. \\
& \left.-\log \left(\left(x_{i}-x_{0}\right)^{2}+\gamma^{2}\right)\right] .
\end{aligned}
$$

The respective score equations are given by

$$
\begin{aligned}
& \frac{\partial l(\Theta)}{\partial x_{0}}=\sum_{i=1}^{n}\left[\frac{2 \lambda \gamma}{\left[\gamma^{2}+\left(x_{i}-x_{0}\right)^{2}\right]\left[\pi-2 \lambda \arctan \left(\frac{x_{i}-x_{0}}{\gamma}\right)\right]}+\frac{2\left(x_{i}-x_{0}\right)}{\left(x_{i}-x_{0}\right)^{2}+\gamma^{2}}\right], \\
& \frac{\partial l(\Theta)}{\partial \gamma}=\frac{n}{\gamma}+\sum_{i=1}^{n}\left[\frac{2 \lambda\left(x_{i}-x_{0}\right)}{\left[\gamma^{2}+\left(x_{i}-x_{0}\right)^{2}\right]\left[\pi-2 \lambda \arctan \left(\frac{x_{i}-x_{0}}{\gamma}\right)\right]}\right. \\
& \left.-\frac{2 \gamma}{\left(x_{i}-x_{0}\right)^{2}+\gamma^{2}}\right] \text {, } \\
& \text { and } \frac{\partial l(\Theta)}{\partial \lambda}=\sum_{i=1}^{n}\left[\frac{-2 \arctan \left(\frac{x_{i}-x_{0}}{\gamma}\right)}{\pi-2 \lambda \arctan \left(\frac{x_{i}-x_{0}}{\gamma}\right)}\right] \text {. }
\end{aligned}
$$

Let $\hat{x_{0}}, \hat{\gamma}$ and $\hat{\lambda}$ be the maximum likelihood estimates of the unknown parameters $x_{0}, \gamma$ and $\lambda$ respectively. We can numerically solve the system of equations $\frac{\partial l(\Theta)}{\partial x_{0}}=0, \frac{\partial l(\Theta)}{\partial \gamma}=0$ and $\frac{\partial l(\Theta)}{\partial \lambda}=0$ to estimate the unknown parameters $x_{0}, \gamma$ and $\lambda$ respectively.

\section{Hazard Rate Function and Reversed Hazard Rate Function}

The survival function or the reliability function $R(t)$ is the probability of an item not failing prior to some time $t$, and the survival function is defined by

$$
R(t)=1-F(t)
$$

where $F(\cdot)$ is the CDF of the transmuted Cauchy distribution defined by equation (5).

The hazard rate function of a random variable $X$ with $\operatorname{PDF} f(x)$ and $\operatorname{CDF} F(x)$ is defined by

$$
h(t)=\frac{f(t)}{1-F(t)} .
$$


Substituting equations (5) and (6) into equation (15), the hazard rate function of the transmuted Cauchy distribution can be expressed as

$$
h(t)=\frac{\frac{\gamma}{\pi^{2}\left[\left(t-x_{0}\right)^{2}+\gamma^{2}\right]}\left[\pi-2 \lambda \arctan \left(\frac{t-x_{0}}{\gamma}\right)\right]}{\frac{1}{2}-\frac{\pi}{4}+\frac{\lambda}{\pi^{2}}\left[\arctan \left(\frac{t-x_{0}}{\gamma}\right)\right]^{2}-\frac{1}{\pi} \arctan \left(\frac{t-x_{0}}{\gamma}\right)} .
$$

By Desai et al. [20], the reversed hazard rate function is a useful tool in the area of maintenance management, particularly for condition monitoring. Its typical behaviour makes it suitable for the assessment of waiting time and hidden failures. The reversed hazard rate function is defined by the expression

$$
r(t)=\frac{f(t)}{F(t)} .
$$

By substituting equations (5) and (6) into equation (17), the reversed hazard rate function of the transmuted Cauchy distribution can be expressed as

$$
r(t)=\frac{\frac{\gamma}{\pi^{2}\left[\left(t-x_{0}\right)^{2}+\gamma^{2}\right]}\left[\pi-2 \lambda \arctan \left(\frac{t-x_{0}}{\gamma}\right)\right]}{-\frac{\lambda}{\pi^{2}}\left[\arctan \left(\frac{t-x_{0}}{\gamma}\right)\right]^{2}+\frac{1}{\pi} \arctan \left(\frac{t-x_{0}}{\gamma}\right)+\frac{\lambda}{4}+\frac{1}{2}} .
$$

\section{Simulation Study}

In this section, the maximum likelihood estimate is evaluated via a simulation study wherein multiple sets of parameters and sample sizes are tested. The location parameter, $x_{0}$, takes the values $-1,-0.05,0$, and 25 , the scale parameter, $\gamma$, takes the values $0.75,1$, and 5 , and the transmutation parameter, $\lambda$, takes the values $-1,-0.5,0,0.5$, and 1. In total there are six parameter combinations are used in simulation study. Sample sizes of $n=50,100$, and 500 are considered. The simulation is repeated 200 times for each combination of parameters and sample size. The estimates and their standard deviations are presented in Table 1. From this table, we see that the expected numerical convergence of the parameters and their standard deviations converging to zero. 


\begin{tabular}{llllllllll}
\hline Sample Size & \multicolumn{3}{l}{ Actual Values } & \multicolumn{3}{l}{ ML Estimates } & \multicolumn{3}{c}{ Standard Deviations } \\
$\mathrm{n}$ & $x_{0}$ & $\gamma$ & $\lambda$ & $\hat{x}_{0}$ & $\hat{\gamma}$ & $\hat{\lambda}$ & $\hat{x}_{0}$ & $\hat{\gamma}$ & $\hat{\lambda}$ \\
\hline 50 & 0 & 1 & 0 & 0.0058 & 1.0156 & 0.0074 & 0.3336 & 0.2369 & 0.3840 \\
& 0 & 1 & -1 & 0.0425 & 0.9617 & -0.9382 & 0.2177 & 0.1757 & 0.1347 \\
& 0 & 1 & 1 & -0.0732 & 0.9996 & 0.9114 & 0.2336 & 0.2252 & 0.1671 \\
& -1 & 1 & 1 & -1.0291 & 0.9794 & 0.9501 & 0.1963 & 0.1982 & 0.1007 \\
& -0.05 & 0.75 & 0.5 & -0.0641 & 0.7922 & 0.5024 & 0.2714 & 0.1744 & 0.3733 \\
& 25 & 5 & -0.5 & 25.0682 & 5.0847 & -0.4869 & 1.4626 & 1.0891 & 0.3178 \\
100 & 0 & 1 & 0 & -0.0303 & 1.0014 & -0.03197 & 0.2405 & 0.1551 & 0.2708 \\
& 0 & 1 & -1 & 0.0172 & 1.0027 & -0.9573 & 0.1356 & 0.1425 & 0.0755 \\
& 0 & 1 & 1 & -0.0363 & 0.9767 & 0.9582 & 0.1260 & 0.1348 & 0.0740 \\
& -1 & 1 & 1 & -1.0269 & 0.9845 & 0.9594 & 0.1321 & 0.1366 & 0.0788 \\
& -0.05 & 0.75 & 0.5 & -0.0567 & 0.7559 & 0.5193 & 0.1710 & 0.1207 & 0.2648 \\
& 25 & 5 & -0.5 & 25.1590 & 4.9935 & -0.4579 & 1.1737 & 0.7595 & 0.2801 \\
500 & 0 & 1 & 0 & 0.0034 & 1.0060 & 0.0080 & 0.1031 & 0.0645 & 0.1208 \\
& 0 & 1 & -1 & 0.0075 & 0.9886 & -0.9848 & 0.0576 & 0.0590 & 0.0265 \\
& 0 & 1 & 1 & -0.0110 & 0.9925 & 0.9849 & 0.0581 & 0.0639 & 0.0267 \\
& -1 & 1 & 1 & -1.0065 & 0.9949 & 0.9840 & 0.0517 & 0.0576 & 0.0280 \\
& -0.05 & 0.75 & 0.5 & -0.0466 & 0.7548 & 0.4994 & 0.0714 & 0.0451 & 0.1067 \\
& 25 & 5 & -0.5 & 24.9792 & 4.9715 & -0.4958 & 0.4491 & 0.3534 & 0.1103 \\
\hline
\end{tabular}

Table 1. ML Estimates and Standard Deviations

\section{Applications}

In this section, we apply the transmuted Cauchy distribution to two real data sets. The first data set is the strength of glass fibers, which originates from the National Physical Lab in England, and the second data set is annual maximum one-day rainfall at Florida Atlantic University.

\subsection{Strength of Glass Fibers}

In this subsection, we fit the transmuted Cauchy distribution to the set of data concerning the strength of the 1.5 $\mathrm{cm}$ glass fibers which is obtained from Smith et al. [30].

\begin{tabular}{llllll}
\multicolumn{6}{c}{ Strengths of $1.5 \mathrm{~cm}$ glass fibers. } \\
\hline 0.55 & 0.74 & 0.77 & 0.81 & 0.84 & 0.93 \\
1.04 & 1.11 & 1.13 & 1.24 & 1.25 & 1.27 \\
1.28 & 1.29 & 1.30 & 1.36 & 1.39 & 1.42 \\
1.48 & 1.48 & 1.49 & 1.49 & 1.50 & 1.50 \\
1.51 & 1.52 & 1.53 & 1.54 & 1.55 & 1.55 \\
1.58 & 1.59 & 1.60 & 1.61 & 1.61 & 1.61 \\
1.61 & 1.62 & 1.62 & 1.63 & 1.64 & 1.66 \\
1.66 & 1.66 & 1.67 & 1.68 & 1.68 & 1.69 \\
1.70 & 1.70 & 1.70 & 1.73 & 1.76 & 1.77 \\
1.78 & 1.81 & 1.82 & 1.84 & 1.84 & 1.89 \\
2.00 & 2.01 & 2.24 & & \\
\hline \multicolumn{7}{l}{ Table 2.5 Strengths of $1.5 \mathrm{~cm}$ glass fibers }
\end{tabular}


The data set, consisting of 63 observations presented in Table 2 has been analyzed in several papers. BarretoSouza et al. [12] analyzed the data using the beta-generalized-exponential distribution. Their paper reported the parameter estimates and the maximized log-likelihood values for the beta-exponential and the beta-generalized exponential distributions. Alzaatreh et al. [6] did the same with the Two-parameter gamma-normal and Fourparameter gamma-normal distributions. The $\beta$-Birnbaum-Saunders and Birnbaum-Saunders were used by Cordeiro et al. [19]. Alshawarbeh et al. [3] used the $\beta$-Cauchy and Cauchy distributions to analyze the data.

\begin{tabular}{|c|c|c|c|}
\hline Distribution & Birnbaum-Saunders & Beta B-S & Beta-Exponential \\
\hline \multirow[t]{4}{*}{ Parameter Estimates } & $\hat{a}=0.2699(0.0267)$ & $\hat{\alpha}=0.3638(0.0709)$ & $\hat{\alpha}=17.7786$ \\
\hline & $\hat{b}=1.3909(0.0521)$ & $\hat{\beta}=7857.566(3602.2)$ & $\hat{\beta}=22.7222$ \\
\hline & & $\hat{a}=1.0505(0.0101)$ & $\hat{\lambda}=0.3898$ \\
\hline & & $\hat{b}=30.4783(0.5085)$ & \\
\hline Log-Likelihood & -22.1890 & -14.7760 & -24.1270 \\
\hline AIC & 48.3780 & 37.5520 & 52.2540 \\
\hline K-S & 0.1988 & 0.1590 & 0.1846 \\
\hline K-S p-value & 0.0355 & 0.1518 & 0.0273 \\
\hline Distribution & Beta Gen. Exp. & 2-param. $\gamma$-normal & 4-param. $\gamma$-normal \\
\hline \multirow[t]{4}{*}{ Parameter Estimates } & $\hat{\alpha}=0.4125$ & $\hat{\alpha}=18.0840(3.5490)$ & $\hat{\alpha}=0.5872(0.7805)$ \\
\hline & $\hat{\beta}=93.4655$ & $\hat{\beta}=0.1459(0.0290)$ & $\hat{\beta}=0.1871(0.5139)$ \\
\hline & $\hat{a}=22.6124$ & & $\hat{\mu}=2.0895(0.7495)$ \\
\hline & $\hat{\lambda}=0.9227$ & & $\hat{\sigma}=0.3910(0.3872)$ \\
\hline Log-Likelihood & -15.5995 & -15.0551 & -13.2148 \\
\hline AIC & 39.1990 & 34.1102 & 34.4296 \\
\hline K-S & 0.1356 & 0.1627 & 0.1620 \\
\hline K-S p-value & 0.1970 & 0.1343 & 0.1377 \\
\hline Distribution & Cauchy & Beta-Cauchy & Transmuted-Cauchy \\
\hline \multirow[t]{4}{*}{ Parameter Estimates } & $\hat{\gamma}=1.5966(0.0248)$ & $\hat{\alpha}=2.4345(1.2411)$ & $\hat{x}_{0}=1.6777(0.0374)$ \\
\hline & $\hat{\lambda}=0.1341(0.0235)$ & $\hat{\beta}=4.0276(2.3276)$ & $\hat{\gamma}=0.1492(0.0291)$ \\
\hline & & $\hat{\theta}=1.7053(0.0783)$ & $\hat{\lambda}=0.9055(0.2807)$ \\
\hline & & $\hat{\lambda}=0.2993(0.1208)$ & \\
\hline Log-Likelihood & -17.6365 & -11.8172 & -14.6063 \\
\hline AIC & 39.2730 & 31.6344 & 35.2127 \\
\hline $\mathrm{K}-\mathrm{S}$ & 0.1029 & 0.0573 & 0.07818 \\
\hline K-S p-value & 0.5167 & 0.9858 & 0.836 \\
\hline
\end{tabular}

Table 3. Parameter estimates (standard errors in parentheses) for the glass fibers data

The model selection is based on the measures of Akaike information criterion (AIC) and Kolmogorov-Smirnov (K-S) test statistics:

$$
A I C=-2 l(\hat{\Theta})+2 q, \quad K S=\max _{1 \leq i \leq N}\left(F\left(Z_{i}\right)-\frac{i-1}{N}, \frac{i}{N}, F\left(Z_{i}\right)\right)
$$

where $q$ is the number of parameters in the model, $l(\hat{\Theta})$ denotes the log-likelihood function evaluated at the maximum likelihood estimates, $F($.$) is the \mathrm{CDF}(5)$, and $Z_{1}, Z_{2}, \ldots, Z_{N}$ are $N$ ordered data points from empirical cumulative distribution function. Here we note that the smaller the values of goodness-of-fit measures the better the fit of the data. The transmuted Cauchy distribution is fitted to the data set. The results are compared to the betaexponential, the beta-generalized exponential, the Two-parameter gamma-normal and Four-parameter gammanormal, $\beta$-Birnbaum-Saunders and Birnbaum-Saunders, $\beta$-Cauchy and Cauchy distributions. The log-likelihood, AIC, and K-S statistics are shown in Table 3. 
Among the distributions with two or three degrees of freedom the transmuted-Cauchy distribution has the largest log-likelihood as well as the second smallest AIC and smallest K-S statistic. These results indicate that the transmuted-Cauchy distribution provides a better fit than the other distributions. However, the Beta-Cauchy distribution, which has four degrees of freedom, has a greater log-likelihood and smaller AIC and K-S statistic.

\subsection{Annual Maximum One-Day Rainfalls at Florida Atlantic University}

The Cauchy distribution can be applied to extreme events such as annual maximum one-day rainfalls and river discharges. Here we analyze data from PRISM Climate Group, Oregon State University [27], which has time series values for individual locations around the world. In particular, we look at the annual maximum one-day rainfalls at Florida Atlantic University as shown in Table 4.

\begin{tabular}{llll}
\hline Year & $\begin{array}{l}\text { Maximum One-Day } \\
\text { Rainfall }(\mathrm{mm})\end{array}$ & Year & $\begin{array}{l}\text { Maximum One-Day } \\
\text { Rainfall }(\mathrm{mm})\end{array}$ \\
\hline 1981 & 80.02 & 2001 & 92.02 \\
1982 & 72.48 & 2002 & 55.01 \\
1983 & 72.17 & 2003 & 57.79 \\
1984 & 102.57 & 2004 & 65.39 \\
1985 & 67.41 & 2005 & 126.03 \\
1986 & 73.43 & 2006 & 73.64 \\
1987 & 52.91 & 2007 & 128.05 \\
1988 & 45.69 & 2008 & 96.73 \\
1989 & 30.3 & 2009 & 117.92 \\
1990 & 35.24 & 2010 & 54.07 \\
1991 & 66.91 & 2011 & 80.02 \\
1992 & 79.85 & 2012 & 87.68 \\
1993 & 47.4 & 2013 & 133.27 \\
1994 & 129.73 & 2014 & 56.29 \\
1995 & 154.54 & 2015 & 64.2 \\
1996 & 59.91 & 2016 & 109.44 \\
1997 & 89.63 & 2017 & 216.63 \\
1998 & 135.05 & 2018 & 63.9 \\
1999 & 102.39 & 2019 & 201.35 \\
2000 & 110.75 & & \\
\hline
\end{tabular}

Table 4. Annual Maximum One-Day Rainfalls at Florida Atlantic University

The transmuted-Cauchy distribution is fitted to the data. The results are compared then to the Cauchy, GammaCauchy, Two-parameter Power Cauchy, and Beta-Cauchy distributions. The log-likelihood, AIC, and K-S statistics are shown in Table 5.

When compared to the Cauchy distribution, the transmuted-Cauchy distribution has the greater log-likelihood as well as the smaller AIC and K-S statistics. These results indicate that the transmuted-Cauchy distribution provides a better fit than Cauchy distribution. When compared to the Beta-Cauchy and Gamma-Cauchy, we see both have greater log-likelihood as well as the smaller AIC which is to be expected because they each have four degrees of freedom rather than three. The histogram in Figure (5) shows the fit of the transmuted-Cauchy distribution. Moreover, one can see the heavy tail characteristic of a Cauchy distribution, but the right-skew of the data shows the need for the transmutation. 


\begin{tabular}{lll}
\hline Distribution & Cauchy & Transmuted-Cauchy \\
\hline \multirow{2}{*}{ Parameter Estimates } & $\hat{x_{0}}=74.8267(5.1193)$ & $\hat{x_{0}}=61.5228(13.9836)$ \\
& $\hat{\gamma}=19.9282(4.3623)$ & $\hat{\gamma}=19.9593(5.6711)$ \\
Log-likelihood & -202.1967 & $\hat{\lambda}=-1.0000(1.14393)$ \\
AIC & 404.3934 & -197.8307 \\
K-S & 0.13966 & 401.6614 \\
K-S p-value & 0.4322 & 0.084705 \\
\hline Distribution & Beta-Cauchy & 0.9423 \\
\hline & $\hat{\theta}=-15.2263(27.5761)$ & $\hat{x}_{0}=3.3334(38.9298)$ \\
Parameter Estimates & $\hat{\lambda}=16.0911(23.5988)$ & $\hat{\gamma}=26.6606(67.5995)$ \\
& $\hat{\alpha}=137.6780(226.4303)$ & $\hat{\alpha}=30.0402(69.8533)$ \\
Log-likelihood & $\hat{\beta}=8.0145(4.8898)$ & $\hat{\beta}=0.0755(0.0895)$ \\
AIC & -194.0601 & -194.0745 \\
K-S & 396.1210 & 396.1489 \\
K-S p-value & 0.071452 & 0.067363 \\
\hline Distribution & 0.9886 & 0.9944 \\
\hline \multirow{3}{*}{ Parameter Estimates } & $\hat{T}$ wo-Parameter Power Cauchy & \\
& $\hat{x_{0}}=0$ & \\
Log-likelihood & $\hat{\alpha}=3.3770$ & \\
AIC & -194.9659 & \\
K-S & 395.9391 & \\
K-S p-value & 0.076543 & \\
\hline
\end{tabular}

Table 5. Parameter estimates (standard errors in parentheses) for Annual Maximum One-Day Rainfalls at Florida Atlantic University

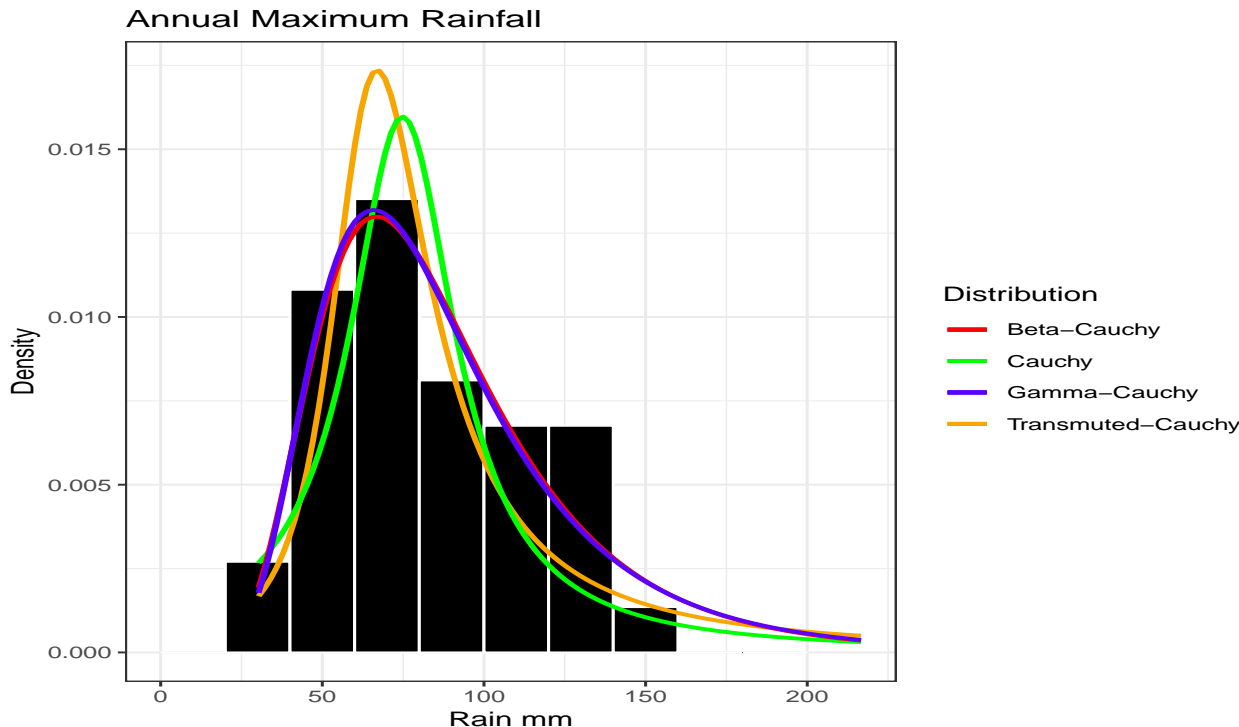

Figure 5. Histogram and Fitted Distribution for Annual Maximum One-Day Rainfall at Florida Atlantic University 


\section{Conclusion}

In this article, we have proposed a new distribution, so-called the transmuted Cauchy distribution (TCD). We have used the Cauchy distribution as the baseline distribution and the quadratic rank transmuted map (QRTM) to construct the transmuted Cauchy distribution. A simulation study is used to assess the performance of the maximum likelihood estimation of the parameters. We also analyzed two real data sets to show the importance and versatility of the proposed model. Various statistics show that the transmuted Cauchy distribution is superior to its competitive models. We hope that this work will serve as a reference for future research in the subject area.

\section{Acknowledgement}

We would like to thank the anonymous reviewers for careful reading of our manuscripts and providing valuable feedback which certainly improved the quality of the paper.

\section{Appendix}

Here we list the probability density function and cumulative distribution function of all distributions that we used in model comparison.

Let $X$ be a normal random variable with the $\operatorname{PDF} \phi(x)$ and the $\operatorname{CDF} \Phi(x)$.

1. Birnbaum-Saunders distribution $B S(\alpha, \beta)$

(a) PDF: $f(x ; \alpha, \beta)=\kappa(\alpha, \beta) x^{-\frac{3}{2}}(x+\beta) \exp \left\{-\frac{\tau(x / \beta)}{2 \alpha^{2}}\right\}, \quad x>0$

(b) $\mathrm{CDF}: F(x ; \alpha, \beta)=\Phi(v), \quad v=\alpha^{-1} \rho(x / \beta), \quad \rho(z)=z^{\frac{1}{2}}-z^{-\frac{1}{2}}$

where $\kappa(\alpha, \beta)=\exp \left(\alpha^{-2} /(2 \alpha \sqrt{2 \alpha \beta})\right.$ and $\tau(z)=z+z^{-1}$.

2. $\beta$ - Birnbaum-Saunders distribution $\beta$ - $B S(\alpha, \beta, a, b)$

(a) PDF: $f(x ; \alpha, \beta, a, b)=\frac{\kappa(\alpha, \beta)}{B(a, b)} x^{-\frac{3}{2}}(x+\beta) \exp \left\{-\frac{\tau(x / \beta)}{2 \alpha^{2}}\right\} \Phi(v)^{a-1}(1-\Phi(v))^{b-1}, \quad x>0$

(b) CDF: $F(x ; \alpha, \beta, a, b)=I_{\Phi(v)}(a, b)$, where $I_{y}(a, b)=\frac{B_{y}(a, b)}{B(a, b)}, B(a, b)=\frac{\gamma(a) \gamma(b)}{\gamma(a+b)}$, $\Phi($.$) is the standard$ cumulative function, $a>0, b>0, v=\alpha^{-1} \rho\left(\frac{t}{\beta}\right), \rho(z)=z^{\frac{1}{2}}+z^{\frac{-1}{2}}$.

3. Beta-exponential distribution $B E(a, b, \lambda)$

(a) PDF: $f(x ; a, b, \lambda)=\frac{\lambda}{B(a, b)} \exp (-b \lambda x)(1-\exp (-\lambda x))^{a-1}$

(b) CDF : $F(x ; a, b, \lambda)=I_{1-\exp -\lambda x}(a, b), \quad x>0, a>0, b>0, \lambda>0$

where $I_{y}(a, b)=\frac{B_{y}(a, b)}{B(a, b)}, B_{y}(a, b)=\int_{0}^{y} w^{a-1}(1-w)^{b-1} d w$.

4. Beta generalized exponential distribution $B G E(a, b, \lambda, \alpha)$

(a) PDF: $f(x ; a, b, \lambda, \alpha)=\frac{\alpha \lambda}{B(a, b)} \exp (-\lambda x)(1-\exp (-\lambda x))^{\alpha a-1}\left\{1-\left(1-\exp (-\lambda x)^{\alpha}\right\}^{b-1}\right.$

(b) $\mathrm{CDF}: F(x ; a, b, \lambda, \alpha)=\frac{1}{B(a, b)} \int_{0}^{\left(1-e^{-\lambda x}\right)^{\alpha}} w^{a-1}(1-w)^{b-1} d w$,

where $x>0, a>0, b>0, \lambda>0, \alpha>0$.

5. Four-parameter gamma normal distribution

(a) PDF: $G(x ; \alpha, \beta, \mu, \sigma)=\frac{\gamma\left(\alpha, \frac{-\log (1-\Phi(x))}{\beta}\right)}{\Gamma(\alpha)}$

(b) CDF: $g(x ; \alpha, \beta, \mu, \sigma)=\frac{1}{\gamma(\alpha) \beta^{\alpha}} \phi(x)[-\log (1-\Phi(x))]^{\alpha-1}[1-\Phi(x)]^{\frac{1}{\beta}-1}$

where $-\infty<x<\infty,-\infty<\mu<\infty, \alpha, \beta, \sigma>0$.

6. Two-parameter gamma normal distribution 
The two-parameter gamma normal is also known as the gamma -standard normal. We obtain the CDF and PDF of the two-parameter gamma normal by replacing $\mu=0$ and $\sigma=1$ in the CDF and PDF of the fourparameter gamma normal distribution.

7. Beta-Cauchy distribution:

(a) PDF: $f\left(x ; x_{0}, a, b, \lambda\right)=\frac{\lambda}{\pi B(a, b)}\left[\frac{1}{2}+\frac{1}{\pi} \arctan \left(\frac{x-x_{0}}{\lambda}\right)\right]^{a-1}\left[\frac{1}{2}-\frac{1}{\pi} \arctan \left(\frac{x-x_{0}}{\lambda}\right)\right]^{b-1} \frac{1}{\lambda^{2}+\left(x-x_{0}\right)^{2}}$

(b) $\mathrm{CDF}: G\left(x ; \alpha, \beta, \gamma, x_{0}\right)=\frac{1}{B(\alpha, \beta)}\left[\frac{1}{\pi} \arctan \left(\frac{x-x_{0}}{\gamma}\right)+\frac{1}{2}\right]^{\alpha-1}\left[\frac{1}{2}-\frac{1}{\pi} \arctan \left(\frac{x-x_{0}}{\gamma}\right)\right]^{\beta-1}$

where $-\infty<x, x_{0}<\infty, 0<a, b, \lambda<\infty$.

8. Gamma-Cauchy distribution:

(a) PDF: $f\left(x ; x_{0}, a, b, \lambda\right)=\frac{\left[-\log \left(\frac{1}{2}-\frac{1}{\pi} \arctan \left(\frac{x-x_{0}}{\lambda}\right)\right)\right]^{a-1}\left[\frac{1}{2}-\frac{1}{\pi} \arctan \left(\frac{x-x_{0}}{\lambda}\right]^{\frac{1}{b}-1}\right.}{\pi \lambda b^{a} \Gamma(a)\left(1+\left(\frac{x-x_{0}}{\lambda}\right)^{2}\right)}$

(b) $\mathrm{CDF}: F\left(x ; x_{0}, a, b, \lambda\right)=\frac{1}{\Gamma(a)} \gamma\left(a,-\frac{1}{b} \log \left(\frac{1}{2}-\frac{1}{\pi} \arctan \left(\frac{x-x_{0}}{\lambda}\right)\right)\right.$

where $\gamma(a, x)=\int_{0}^{x} t^{a-1} e^{-t} d t,-\infty<x, x_{0}<\infty, 0<a, b, \lambda<\infty$.

9. Power Cauchy distribution:

(a) $\operatorname{PDF}: f\left(x ; x_{0}, a, \lambda\right)=\frac{2 a\left(\frac{x-x_{0}}{\lambda}\right)^{a-1}}{\pi \lambda\left(1+\left(\frac{x-x_{0}}{\lambda}\right)^{2 a}\right)}$

(b) $\mathrm{CDF}: F\left(x ; x_{0}, a, \lambda\right)=\frac{2}{\pi} \arctan \left(\frac{x-x_{0}}{\lambda}\right)^{a}$

References

1. M. Alizadeh, M. Emadi, and M. Doostparast (2019). A New Two-Parameter Lifetime Distribution: Properties, Applications and Different Method of Estimations, Statistics, Optimization \& Information Computing, vol. 7, 291-310.

2. M. Aljarrah, C. Lee, and F. Famoye (2014). On generating T-X family of distributions using quantile functions. J. Stat. Distrib. App., $15(2)$.

3. E. Alshawarbeh, F. Famoye, and C. Lee (2013). Beta-Cauchy distribution: some properties and applications. Journal of Statistical Theory and Applications, 12(4):378-391.

4. R. Alshkaki (2020). A Generalized Modification of the Kumaraswamy Distribution for Modeling and Analyzing Real-Life Data. Statistics, Optimization \& Information Computing, 8(2), 521-548.

5. A. Alzaatreh, C. Lee, and F. Famoye (2013). A new method for generating families of continuous distributions. Metron, 71(1), 63-79.

6. A. Alzaatreh, F. Famoye, and C. Lee (2014). The gamma-normal distribution: Properties and applications. Computational Statistics \& Data Analysis, 69:67-80.

7. A. Alzaatreh, C. Lee, F. Famoye, and I. Ghosh (2016). The generalized Cauchy family of distributions with applications. J. Stat. Distrib. App., 3(12).

8. G. Aryal, and C. Tsokos (2009). On the transmuted extreme value distribution with application. Nonlinear Analysis: Theory, Methods and Applications, 71:1401-1407.

9. GR Aryal (2013). Transmuted Log-Logistic Distribution. Journal of Statistics Applications \& Probability, 2(1): 11-20.

10. G. Aryal, (2019). On The Beta Exponential Pareto Distribution. Statistics, Optimization \& Information Computing, 7(2), 417-438.

11. S. Ashour, and M. Eltehiwy (2013). Transmuted Lomax distribution. American Journal of Applied Mathematics and Statistics, 1(6): 121-127.

12. W. Barreto-Souza, A.H. Santos, and G. M. Cordeiro (2010). The beta generalized exponential distribution. Journal of Statistical Computation and Simulation, 80(2):159-172.

13. R. Carrillo, T. Aysal, and K. Barner (2010). A generalized Cauchy distribution framework for problems requiring robust behavior EURASIP J. Adv. Signal Process, (312989).

14. C. Chesneau, H. S. Bakouch, and M. N. Khan, (2020). A weighted transmuted exponential distribution with environmental applications. Statistics, Optimization \& Information Computing, 8(1), 36-53.

15. S. Chhetri, A. Akinsete, G. Aryal, and H. Long (2017). The Kumaraswamy transmuted Pareto distribution. J. Stat. Distrib. App., $4(11)$.

16. S. B. Chhetri, H. Long, and G. Aryal (2017). The Beta Transmuted Pareto Distribution: Theory and Applications. Journal of Statistics Applications \& Probability, 6(2), 243-258.

17. G. M. Cordeiro, and A. J. Lemonte (2011). The Beta-Half-Cauchy distribution. Journal of Probability and Statistics, 1-18.

18. G. M. Cordeiro, and M. D.Castro (2011). A new family of generalized distributions. Journal of Statistical Computation and Simulation, 81(7):883-898.

19. G. M. Cordeiro, and A. J. Lemonte (2011). The $\beta$-BirnbaumCSaunders distribution: an improved distribution for fatigue life modeling. Computational Statistics \& Data Analysis, 55(3):1445-1461.

20. D. Desai, V. Mariappan, and M. Sakhardande (2011). Nature of reversed hazard rate: an investigation. Int J Perform Eng, 7(2):165171. 
21. I. Elbatal, and G. Aryal (2015). Transmuted Dagum distribution with applications. Chilean J. of Statistics, 6(2):31-45.

22. M. Kateregga, S. Mataramvura, and D. Taylor (2017). Parameter estimation for stable distributions with application to commodity futures log-returns. Cogent Economics \& Finance, 5(1).

23. M. Khan, R. King, and I. Hudson (2016). Transmuted Kumaraswamy distribution. Stat. Transit., 17(2):183-210.

24. F. Merovci, and L. Puka (2014). Transmuted Pareto distribution. ProbStat. Forum, 7:1-11.

25. S. Nadarajah, and S. Kotz (2007). A skewed truncated Cauchy distribution with applications in economics, Applied Economics Letters, 14:13, 957-961, DOI: 10.1080/13504850600705950

26. M. Pal, and M. Tiensuwan (2014). The beta transmuted Weibull distribution. Austrian Journal of Statistics, 43(2):133-149.

27. PRISM Climate Group, Oregon State University (2019). Time series values for individual locations. http://prism.oregonstate.edu, Created: 2019-11-10.

28. W. Shaw, and I. Buckley (2007). The alchemy of probability distributions: beyond Gram-Charlier expansions, and a skew-kurtoticnormal distribution from a rank transmutation map. Research report, pages 2760-2778.

29. W. Shaw, and I. Buckley (2009). The alchemy of probability distributions: beyond Gram-Charlier expansions, and a skew-kurtoticnormal distribution from a rank transmutation map. Conference on Computational Finance, IMA, 0901C0434, Research report.

30. R. L. Smith, and J. C. Naylor (1987). A comparison of maximum likelihood and Bayesian estimators for the three- parameter Weibull distribution. Journal of the Royal Statistical Society. Series C (Applied Statistics), 36(3):358-369. 